\title{
A SIMPLE MODEL TO QUANTIFY ROCKING ISOLATION
}

\author{
Sinan Acikgoz ${ }^{1}$ and Matthew J. DeJong ${ }^{2}$
}

(Submitted August 2016; Reviewed March 2017; Accepted July 2017)

\begin{abstract}
Rocking action at the foundation-structure interface has long been proposed to isolate structures from strong ground motion. In this paper, the fundamental concept of rocking isolation is examined in depth to guide further design efforts. This is achieved by first deriving an analytical model of a flexible structure freely rocking on rigid ground. Decomposing the coupled equations of motion of the model into their modal components provides new information on the mechanics of rocking isolation. After identifying the salient parameters needed to quantify rocking isolation, equations to predict the lateral accelerations, base shear and overturning moments arising during rocking are provided. The analytical model and the simplified equations are then validated using some of the earliest experiments on rocking structures, which were completed in New Zealand. These validations clarify poorly understood phenomena concerning rocking isolation, such as how rocking and vibrations of the structure couple, how this influences the excitation mechanisms of the structure, resulting in seismic shear forces and overturning moments larger than those required for uplift. The findings provide an analytical basis for designing efficient rocking systems that successfully limit force demands.
\end{abstract}

\section{INTRODUCTION}

Utilizing a rocking mechanism at the interface of the structure and the foundation has the potential to isolate the superstructure [1]. This isolation effect is illustrated in Figure 1, where a pushover test is performed on a single degree of freedom elastic structure resting on ideally rigid ground. Initially, the structure responds to increasing lateral displacements $\Delta$ with a positive structural stiffness. Therefore, a linear relationship is observed between the induced lateral displacements and the corresponding base overturning moment. However, once rocking is initiated, the weight of the structure (instead of elastic structural stiffness) acts as the self-centring force. The total resisting moment provided by the weight decreases as the structure rotates away from the ground, resulting in 'negative stiffness' behaviour (Figure 1). Rocking isolation is expected for two reasons. First, the force required to overturn the structure decreases with increasing rocking angles, resulting in a capping of the maximum static lateral force demand to the force that causes uplift. Second, after uplift occurs, the ground motion couples primarily with the rotation rather than the deformation of the structure. Thus, the dynamic force demand reduces because the vibration mode deformation response is mostly decoupled from the ground, while the lateral force demand due to rocking rotation is limited.

Inspired by these desirable dynamic characteristics, New Zealand practitioners and researchers pioneered the use of rocking for earthquake resistant design in the early 1970s. Some of the earliest published practical applications of rocking mechanisms, the Rangitikei Viaduct Railway Bridge [2] and the Christchurch Rocking Chimney [3], are from this period. Similarly, some of the earliest tests on the use of rocking mechanisms in buildings and bridges were conducted at the University of Canterbury around the same time [4,5]. These pioneering studies inspired decades of research in the field. Most notably, various researchers have noted the low inherent damping associated with rocking action and have conducted studies on increasing the energy dissipation and self-centering capacity of rocking systems with supplemental dampers (e.g. $[6,7])$. More recent efforts have investigated new rocking systems in buildings where only a small proportion of the seismic mass is allowed to rock and drag the rest of the structure [8].

In order to provide a deeper understanding of the dynamics of flexible rocking systems, the authors of this paper have developed new analytical models $[9,10]$. These models confirmed aforementioned aspects of rocking isolation [11,12]. However, they also revealed the important coupling between rocking and vibrations, where the rocking action influenced the vibration characteristics and excitation mechanisms of the structure. Significant changes in vibration mode frequencies and shapes were predicted analytically and observed experimentally. In addition, these studies highlighted the potential for superstructure vibrations to be excited at impact as well as directly by ground motions $[13,14]$. These findings suggest that rocking isolation cannot be sufficiently described by Figure 1, where the coupling between superstructure vibrations, impact and ground motion is neglected.

In the light of these recent studies, the concept of rocking isolation is re-examined in this paper. This is achieved by deriving the nonlinear equations of motion of a flexible structure, freely rocking on rigid ground. These equations are linearized and simplified to identify the salient characteristics of rocking isolation. Then, by using the experimental results in some of the earliest tests on rocking structures by Evison and McManus [4,5], these simplified equations, and the new understanding of rocking isolation they provide, are validated. Further, the results quantify the influence of building slenderness and the transition (i.e. impact) between rocking cycles on rocking isolation. Finally, the paper closes by summarizing the major conclusions from this study. 


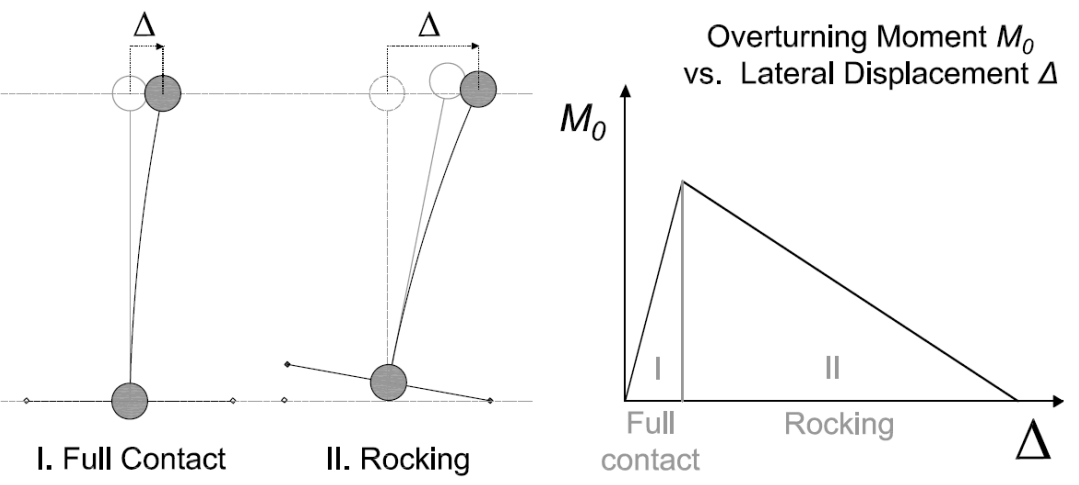

Figure 1: Schematic representation of a displacement controlled pushover test on an idealized flexible rocking structure. Response during (left) full contact and (right) rocking phases, with the corresponding overturning moment and lateral displacement relationship.

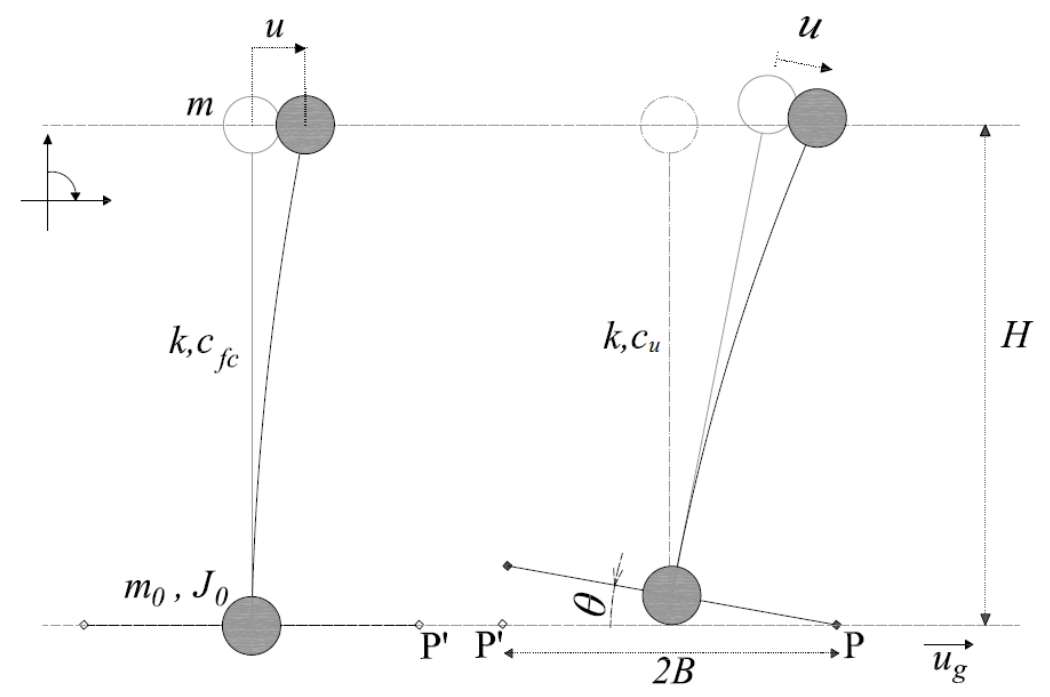

Figure 2: Schematic of the idealized analytical model for flexible rocking structures during full contact and rocking phases.

\section{ANALYTICAL MODEL OF A FREELY ROCKING FLEXIBLE STRUCTURE}

\section{Equations of Motion and Phase Transition}

The analytical model is shown in Figure 2, with two lumped masses, mass $m$ at height $H$, and mass $m o$ at the base which represents the foundation mass. It was demonstrated in an earlier study that this model may describe a wide range of flexible structures and capture the critical coupling between primary superstructure vibrations and rocking [15].

To describe the mass distribution in the structure, a dimensionless constant is defined as $\gamma=\left(m+m_{0}\right) / m$. The masses are connected by an axially rigid strut which can elastically deform parallel to the foundation beam. The stiffness associated to this movement is given by $k$ and for convenience, the structural damping is approximated by the viscous damping coefficient $c_{f c}$. The generalized coordinate $u$ in the rotating frame of reference describes the elastic action, while the other generalized coordinate $\theta$ describes the rocking action. The rocking action is allowed to occur only about the pivot points at the edges of the foundation. During the rocking phase depicted in Figure 2, the structure rocks about the pivot point $\mathrm{P}$ and is assumed to maintain contact with this point during rocking. The impending pivot point is denoted by P'.

$$
\ddot{u}+2 \zeta_{f c} \omega_{f c} \dot{u}+\omega_{f c}^{2} u=-\ddot{u}_{g}
$$

where vibration frequency is described as $\omega_{f c}=\sqrt{k / m}$ and the ratio of critical damping is defined as $\zeta_{f c}=c_{f c} /(2 \sqrt{\mathrm{km}})$ during full contact stage. The base shear $V_{f c}$ and overturning moment $M_{f c}$ during this phase of the motion is given by:

$$
\begin{aligned}
& V_{f c}=m\left(\ddot{u}+\ddot{u}_{g}\right) \\
& M_{f c}=m H\left(\ddot{u}+\ddot{u}_{g}\right)
\end{aligned}
$$

For a structure that is fixed to the ground, the maximum acceleration demand $\left(\ddot{u}+\ddot{u}_{g}\right)$ can be estimated as the spectral demand at the structural vibration period $T_{f c}=\frac{2 \pi}{\omega_{f c}}$ as $S_{a}\left(T_{f c}\right)$. Here, the spectra may refer to a spectra derived from earthquake records or the codified design spectra. This results in a maximum base shear demand estimate $\left(V_{f c}\right)_{\max }$ as follows:

$$
\left(V_{f c}\right)_{\max }=m S_{a}\left(T_{f c}\right)
$$

However, a structure may begin to rock if it is unrestrained. An approximate way to estimate the initiation of rocking is to define the critical displacement, $u_{c r}=\gamma g \tan (\alpha) / \omega_{f c}{ }^{2}$ where $g$ 
is gravity and $\alpha=\tan ^{-1}(B / H)$ is the slenderness of the top mass. When the structure is displaced to this critical value, rocking is initiated as the overturning moment is larger than the resisting moment about one of the pivot points. Using a Lagrangian approach, the equations of motion for the rocking phase can be written as:

$$
\begin{aligned}
m \ddot{u}+ & m H \ddot{\theta}+m( \pm B-u) \dot{\theta}^{2}+c_{u} \dot{u}+k u=-m \ddot{u}_{g} \cos \theta \\
& +m g \sin \theta \\
m H \ddot{u}+ & \left(J_{0}+m\left(R_{0}^{2}+u^{2} \mp 2 B u\right)+m_{0} B^{2}\right) \ddot{\theta} \mp 2 m \dot{\theta} \dot{u}(B \mp u) \\
= & \mp m_{0} \ddot{u}_{g} B \sin \theta+m \ddot{u}_{g}\left(-R_{0} \cos (\alpha \mp \theta)+u \sin \theta\right) \\
& \mp m_{0} g B \cos \theta+m g\left(\mp R_{0} \sin (\alpha \mp \theta)+u \cos \theta\right)
\end{aligned}
$$

where a new viscous damping parameter $c_{u}$ is defined during the rocking stage in order to calibrate structural damping during the rocking phase, $R_{0}=\sqrt{B^{2}+H^{2}}$ is the radial distance from mass $\mathrm{m}$ to the pivot point while the system is at rest and $J_{0}$ is the lumped mass moment of inertia of the foundation beam about the mid-point of the base. Equation (5) describes the force equilibrium in the direction of the rotating coordinate $u$ and Equation (6) describes the moment equilibrium about the pivot point. In these equations, the upper sign describes rocking about the right pivot point and the lower sign about the left pivot point. A similar notation will be used through this paper to describe the piecewise defined equations of motion.

Equations (1) and (5-6) are solved numerically to describe the response during full contact and rocking phases. The transition from a full contact phase to a rocking phase was defined earlier. It is also necessary to define the transition from rocking motion once the structure impacts with the ground. During this transition, energy is dissipated and the motion may continue with a new rocking phase or a full contact phase. It is assumed that if the angular momentum about the impacting corner is in the same direction as angular velocity, then a new rocking phase occurs and the angular velocity is reduced by the coefficient of restitution $r$, while all other displacements and velocities are assumed to remain the same. In the case that the angular moment about the impacting corner and the angular velocity have opposite sign, a full contact motion follows with conservation of horizontal momentum before and after the impact. This is a simple transition model, which makes a number of unverified assumptions concerning the superstructure response during impact.

This simple impact model captures the general characteristics of response and can conservatively estimate acceleration demands. Since the specific value of the coefficient of restitution has limited influence on the maximum accelerations experienced during rocking, empirically determined values are used to investigate experimental data in Section 3. While this modelling approach is sufficient for the investigations carried out in this paper, precise time history prediction of rocking response would require a reliable estimation of behavior at impact, which can rarely be captured with a single coefficient of restitution. To address these issues, more sophisticated impact models (e.g. $[13,16])$ inspired by experimental studies (e.g. $[14,17,18])$ have been proposed, but are beyond the scope of this paper.

\section{Modal Components of Response}

To obtain an intuitive understanding, Equations (5) and (6) can be linearized. As only small rocking and vibration motion would be allowed in an earthquake resistant system, the equations are linearized about their initial $(u=0, \theta=0)$ position. This yields:

$$
\begin{gathered}
\ddot{u}+H \ddot{\theta}+\frac{c_{u}}{m} \dot{u}+\omega_{f c}^{2} u-g \theta=-\ddot{u}_{g} \\
H \ddot{u}+\frac{J_{P}}{m} \ddot{\theta}-g u-g H \theta=-H \ddot{u}_{g} \mp \gamma g B
\end{gathered}
$$

Here, the moment of inertia of the superstructure about the pivot point is given by $J_{P}=J_{0}+m R_{0}^{2}+m_{0} B^{2}$. After expressing Equations (7) and (8) in matrix notation, the following compact expression is obtained:

$$
\begin{gathered}
{\left[\begin{array}{cc}
1 & H \\
H & \frac{J_{p}}{m}
\end{array}\right] \ddot{z}+\left[\begin{array}{cc}
\frac{c_{u}}{m} & 0 \\
0 & 0
\end{array}\right] \dot{\underline{z}}+\left[\begin{array}{cc}
\omega_{f c}^{2} & -g \\
-g & -g H
\end{array}\right] \underline{z}} \\
=\left[\begin{array}{c}
-\ddot{u}_{g} \\
-H \ddot{u}_{g} \mp \gamma g B
\end{array}\right]
\end{gathered}
$$

where the state vector $\underline{z}=[u, \theta]^{T}$ encompasses all the variables in the system and the superscript $T$ denotes the transpose operation. The linearized Equations (9) have a simpler form but they still demonstrate mass coupling. In order to obtain further insight, the response can be decomposed to into its modal components.

Accumulating experimental and analytical evidence [13,14] suggests that the dynamic response of flexible rocking structures is composed of orthogonal modes of response. These modes of response are (i) the weakly coupled rocking mode and (ii) coupled vibration modes (see Figure 3 ). The rocking mode describes the rigid body rotation of the structure as a whole with negligibly small elastic motion. Contrastingly, the vibration mode describes the coupled vibration and base rotation. For the simple two degree of freedom analytical model that is examined herein, only a single vibration mode exists. In order to describe these modal components, the state vector in Equation (9) can be expressed with Rayleigh's method as $\underline{z}=\underline{v} q$, where the eigenvector $\underline{v}$ and generalized coordinate $q$ of an arbitrary mode are introduced.

\section{Rocking Mode}

For the weakly coupled rocking mode, the mode shape can be approximated as $v_{r}=[0,1]^{T}$ and the generalized coordinate is defined as $q_{r}$. Here the subscript $r$ refers to the rocking mode. Since this mode is orthogonal to the vibration mode, premultiplying Equation (9) with $v_{r}^{T}$ results in the decoupled differential equation for the rocking mode:

$$
\frac{J_{p}}{m} \ddot{q}_{r}-g H q_{r}=-H \ddot{u}_{g} \mp \gamma g B
$$




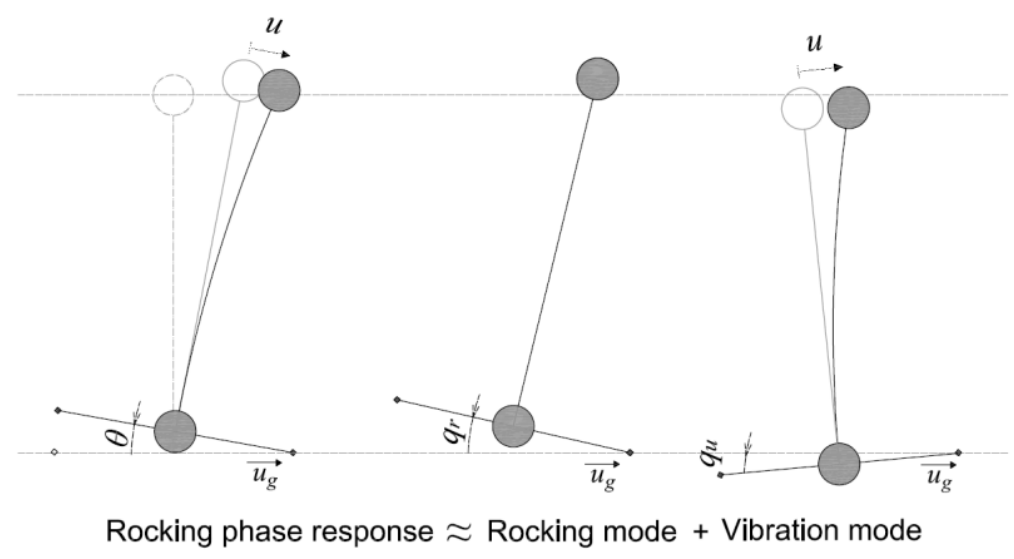

Figure 3: A schematic representation of the modal components of response during rocking phase.

Equation (10) can be further simplified. In analogy with the rigid rocking block formulation of Housner [1], the linearized system frequency parameter may be defined as $p_{l}=\sqrt{m g H / J_{P}}$. This parameter relates to the frequency of a pendulum and is useful for evaluating the effective period of rocking motion. Similarly, the slenderness angle of the center of gravity is defined as $\alpha_{c g}=\tan ^{-1}(B \gamma / H)$. By using these definitions, Equation (10) can be expressed as:

$\ddot{q}_{r}-p_{l}^{2} q_{r}=-p_{l}^{2} \frac{\ddot{u}_{g}}{g} \mp p_{l}^{2} \tan \left(\alpha_{c g}\right)$

The resulting Equation (11) is identical to the equation of motion of a rigid structure with similar geometry and mass, when it is linearized about its rest position. The rocking mode exhibits negative stiffness, which indicates that the force demands due to this component of response decrease with increasing rocking angles. This is demonstrated by $a_{r}$, the lateral acceleration demand at the location of the lumped top mass due to this rocking mode:

$a_{r}=H p_{\iota}^{2}\left(\mp \tan \alpha_{c g}+q_{r}-\frac{\ddot{u}_{g}}{g}\right)$

It is reasonable to assume that rocking action will be small. Therefore the acceleration demand due to the rocking mode can be reliably expressed by substituting $q_{r}=0$ in Equation (12):

$a_{r}=\mp \chi g \tan \alpha_{c g}-\chi \ddot{u}_{g}$

where $\chi=\frac{H p_{l}^{2}}{g}$ is an important dimensionless modal participation term.

In addition, Equation (11) provides information on the periodicity of the rocking motion. The equation has real-valued roots and conjugate eigenvalues of $\pm p_{l}$. Therefore, its homogenous form can be solved using hyperbolic functions. These functions describe motion which has a frequency that decreases with the amplitude of rocking motion. To quantify this relationship, the time it takes structure to impact with the ground after being released from an initial angle $\theta_{i}$, can be determined. This provides a measure of the effective rocking frequency $f_{r}$ as follows:

$$
f_{r}=\frac{p_{l}}{4}\left(\cosh ^{-1}\left(\frac{1}{1-\frac{\theta_{i}}{\tan \alpha_{c g}}}\right)\right)^{-1}
$$

Equation (14) is similar to the rocking period derived by Housner (1963). As expected, Equation (14) assumes the value of zero for $\theta_{i}=0$ and tends to infinity as $\theta_{i}$ approaches $\alpha_{c g}$. It is noteworthy that this effective rocking period only considers the motion due to the rocking mode and does not consider the vibration mode, which will be examined next.

\section{Vibration Mode}

The second component of response is the coupled vibration mode, which includes elastic and rocking components. It is indicated with a subscript $u$. Therefore, its vibration mode shape is made up of the elastic vibrations $u$ and the rocking component $q_{u}$. Since the total rocking angle is given by $\theta=q_{r}+q_{u}$ (see Figure 2), Equation (8) can be expressed as:

$$
\begin{aligned}
\frac{p_{l}^{2}}{g} \ddot{u}+\left(\ddot{q}_{r}+\ddot{q}_{u}\right)-\frac{p_{l}^{2}}{H} u-p_{l}^{2}\left(q_{r}+q_{u}\right)= \\
-p_{l}^{2} \frac{\ddot{u}_{g}}{g} \mp p_{l}^{2} \tan \left(\alpha_{c g}\right)
\end{aligned}
$$

Subtracting Equation (11) from Equation (15) and neglecting the small geometric stiffness terms yields:

$$
\ddot{q}_{u}=-\frac{p_{l}^{2}}{g} \ddot{u}
$$

Equation (16) defines a simple relationship between the elastic vibration and rocking component of this coupled mode. This relationship is used to define the eigenvector of the coupled vibration mode as $\underline{v_{u}}=\left[1,-p_{l}^{2} / g\right]^{T}$ for the generalized coordinate $u$. Pre-multiplying this eigenvector with Equation (9) yields the following modal equation: 


$$
\begin{aligned}
& \left(1-\frac{H p_{l}^{2}}{g}\right) \ddot{u}+2 \frac{c_{u}}{m} \dot{u}+\left(\omega_{f c}^{2}+2 p_{l}^{2}-\frac{H p_{l}^{2}}{g} p_{l}^{2}\right) u= \\
& -\ddot{u}_{g}\left(1-\frac{H p_{l}^{2}}{g}\right) \pm \frac{H p_{l}^{2}}{g} g \tan \alpha_{c g}
\end{aligned}
$$

Noting that $p_{l}<<\omega_{f c}$ for most engineering structures, Equation (17) can be simplified. When the vibration frequency and modal damping are defined as $\omega_{u} \approx \omega_{f c} / \sqrt{1-\chi}$ and $\zeta_{u} \approx c_{u} /(2 \sqrt{k m} \sqrt{1-\chi})$, the following single degree of freedom equation is obtained to describe the coupled vibration mode:

$$
\ddot{u}+2 \zeta_{u} \omega_{u} \dot{u}+\omega_{u}^{2} u=-\ddot{u}_{g} \pm \frac{\chi}{1-\chi} g \tan \alpha_{c g}
$$

Contrary to the earlier rocking mode, the vibration mode demonstrates positive stiffness. In general, Equation (18) is similar to Equation (2), the equation of motion during full contact. However, notable differences exist. First, the vibration frequency associated with this mode is $\omega_{u}$. Since $\chi$ is close to but always smaller than unity, $\omega_{u}$ is higher than $\omega_{f c}$. This increase is due to a decrease in the effective mass participating in this vibration mode, as illustrated in Equation (17). The increase will be most significant for slender structures where $\chi$ tends towards unity.

More importantly, a new forcing term $g \tan \alpha_{c g}$ arises in Equation (18) compared to Equation 11 due to the coupling of vibrations and rocking action. This gravity forcing term is strongly affected by the geometry of the structure and the modal contribution factor $\chi /(1-\chi)$. The piecewise definition of the gravity forcing has potential to induce significant vibrations when the structure transitions from one rocking phase to another and the forcing instantaneously changes direction. This effect is expected to be more severe for stocky structures where $\alpha_{c g}$ is larger.

\section{Evaluation of modal lateral acceleration, shear and overturning moment demands}

To evaluate how these findings illuminate rocking isolation, $a_{u}$ , the lateral acceleration demand at height $H$ due to the vibration mode and inertial accelerations, can be examined:

$$
a_{u}=\ddot{u}+\ddot{u}_{g}+H \ddot{q}_{u}=\ddot{u}+\ddot{u}_{g}-\chi \ddot{u}
$$

Summing Equation (19) with the acceleration demands due to the rocking mode (Equation (13)) provides an expression for the total lateral acceleration demand $a_{t}$ at height $H$ :

$$
a_{t}=\mp \chi g \tan \alpha_{c g}+(1-\chi)\left(\ddot{u}+\ddot{u}_{g}\right)
$$

A conservative estimate of maximum lateral accelerations of the structure can be made by summing up the maximum values that are attained for each term in Equation (20). However, to estimate the second term, further simplifications are required. The vibrations due to the ground motion and gravity components in this term can be separated, as $\ddot{u}=\ddot{u}_{g m}+\ddot{u}_{g r}$ where $\ddot{u}_{g m}$ is the direct vibration excitation due to ground motion and $\ddot{u}_{g r}$ is the excitation due to gravity forcing, which is estimated by a force of constant magnitude with reversing polarity at impact (see the last term of Equation (18)).

A simple estimation for $\left(\ddot{u}_{g m}+\ddot{u}_{g}\right)_{\max }$ may be made from the design spectra as $S_{a}\left(T_{u}=2 \pi / \omega_{u}\right)$, which provides an upperbound contribution for the vibrations due to direct excitations from the ground motion. This is clearly a conservative estimation of maximum acceleration due to direct excitation; it is unlikely for consistent amplification of elastic motion similar to a fixed base structure to occur within a short rocking cycle. While a better estimation of the direct excitation of the structure by ground motion during rocking would be useful, the proposed simplification can be used as an upper bound estimate.

In addition, during transition from one rocking cycle to another, the gravity forcing can be described with a step load with a finite rise time, representing the impact duration. Then, for quiescent initial values, the maximum magnitude of induced accelerations due to gravity forcing can be estimated as follows:

$\left(\ddot{u}_{g r}\right)_{\max }=C \chi g \tan \alpha_{c g} /(1-\chi)$

where $C$ is a dynamic amplification factor. In a conservative assumption, the rise time may be taken as zero and damping may be assumed negligible. In this case, $C=2$. If the rise time is long or if the structure is overdamped, then $C$ may be as low as 0 . On the basis of this information, maximum total lateral acceleration, base shear and overturning moment demands $a$, $V$ and $M$, are estimated as follows:

$$
\begin{aligned}
& a_{t}=(1-\chi) S_{a}\left(T_{u}\right)+\chi(C+1) g \tan \alpha_{c g} \\
& V_{t}=m(1-\chi) S_{a}\left(T_{u}\right)+m \chi(C+1) g \tan \alpha_{c g} \\
& M_{t}=m H(1-\chi) S_{a}\left(T_{u}\right)+m H \chi(C+1) g \tan \alpha_{c g}
\end{aligned}
$$

Equations (23) and (24) suggests that the isolation provided by rocking is complex. In these equations, the first term describes the force/moment demand resulting from the inertial accelerations and vibrations caused by the ground accelerations. Typically the values of $\chi$ are close to unity, so the first term is small. Therefore in comparison to elastic systems which are not allowed to uplift, a reduction in base shear and moment demands are likely (compare Equations (4) and (23)). The second term in Equations (23) and (24) describes the force/moment contribution due to: i) the rocking mode and ii) the vibrations due to the change in gravity forcing at the transition between rocking phases. The value of $C$, which relates structural characteristics to the abruptness of the transition and varies from 0 to 2 , provides a clear indication of the contribution of vibrations induced at impact to the total base shear demand. Therefore, the effectiveness of rocking isolation depends on the particular structural and geometric characteristics and needs further examination. This will be illustrated for two experimental structures in the following sections.

\section{EXPERIMENTAL INVESTIGATION}

The modal components of response presented in Section 2 provide insight into the mechanics of how rocking isolates the structure. Experimental proof for the findings can be found in some of the earliest tests on rocking structures, which were completed at the University of Canterbury, by Evison [4] and McManus [5]. These investigate the free vibration and earthquake response of small-scale models of a stiff shear wall 
structure and a bridge pier. On the laboratory scale, both structures were represented by similar lumped mass models. Masses were attached on a steel column which was welded to a stiff and heavy rectangular foundation plate at its base. Adjustable bolts which protrude through the foundation plate were fixed on each corner of the base plate, acting as rocking feet about which the structure pivots.

Figure 4 shows the experimental setup of Evison [4]. The instrumentation, illustrated in Figure 4 (right), is composed of Linear Variable Displacement Transducers (LVDT) and directcurrent accelerometers. The LVDTs are mounted on a fixed steel instrumentation rig. Two LVDTs on either side of the column measure the uplift displacements at the edge of the foundation plate while another LVDT at the tip of the column measures the total horizontal displacement. These are complemented by an accelerometer which measures the total lateral acceleration $a$ at the tip of the column, where the additional $120 \mathrm{~kg}$ mass is lumped. This acceleration may be used to estimate the total base shear and moment and is therefore used as a proxy for force demands in this paper.

Table 1 shows the relevant dimensions and parameters used for the analytical model. The total mass $m_{0}$ of the foundation beam and plate was lumped at zero height and the additional mass $m$ was lumped at $H=1.05 \mathrm{~m}$. The column mass was distributed evenly. No additional inertia $J_{0}$ was lumped at the foundation.
The slenderness angle of the centre of gravity of the structure is defined with $\alpha_{c g}=0.35 \mathrm{rad}$ and the modified frequency parameter is given by $p_{l}=2.92 \mathrm{rad} / \mathrm{s}$. The stiffness was defined to match the experimentally determined first-mode frequency $(11 \mathrm{~Hz})$ whereas the structural damping factor $\left(\zeta_{f c}=1.5 \%\right)$ and the coefficient of restitution ( $r=0.96)$ due to impact were defined empirically to best fit the data. A viscous damping constant of $c_{f c}$ was calculated on the basis of these parameters, and in the absence of further information, it was assumed that $c_{u}=c_{f c}$.

To compare the analytical model to experimental results, the recorded uplift and horizontal displacements (denoted respectively by $\delta_{u}$ and $\delta_{t}$ ) and total lateral acceleration $a$ at the top of the column (in the direction of the rotating coordinate) were expressed as follows:

$$
\begin{aligned}
& \delta_{u}=2 B \sin \theta \\
& \delta_{t}=( \pm B(1-\cos \theta))+u \cos \theta+H \sin \theta \\
& a=\ddot{u}+H \ddot{\theta}+( \pm B-u) \dot{\theta}^{2}
\end{aligned}
$$

Table 1: Important parameters for the analytical model representing the experimental model of Evison [4].

\begin{tabular}{|c|l|}
\hline Parameter & Value \\
\hline$B(\mathrm{~m})$ & 0.285 \\
\hline$H(\mathrm{~m})$ & 1.05 \\
\hline$m(\mathrm{~kg})$ & 125.8 \\
\hline$m_{0}(\mathrm{~kg})$ & 44.02 \\
\hline
\end{tabular}

\begin{tabular}{|c|l|}
\hline Parameter & Value \\
\hline$J_{0}\left(\mathrm{~kg} \cdot \mathrm{m}^{2}\right)$ & 0 \\
\hline$\omega_{f c}(\mathrm{~Hz})$ & 11 \\
\hline$\zeta_{f c}$ & $1.5 \%$ \\
\hline$r$ & 0.96 \\
\hline
\end{tabular}

\begin{tabular}{|c|l|}
\hline Parameter & Value \\
\hline$J_{\mathrm{P}}\left(\mathrm{kg} \cdot \mathrm{m}^{2}\right)$ & 152.5 \\
\hline$\alpha_{c g}(\mathrm{rad})$ & 0.35 \\
\hline$p_{l}(\mathrm{rad} / \mathrm{s})$ & 2.92 \\
\hline$\chi$ & 0.92 \\
\hline
\end{tabular}
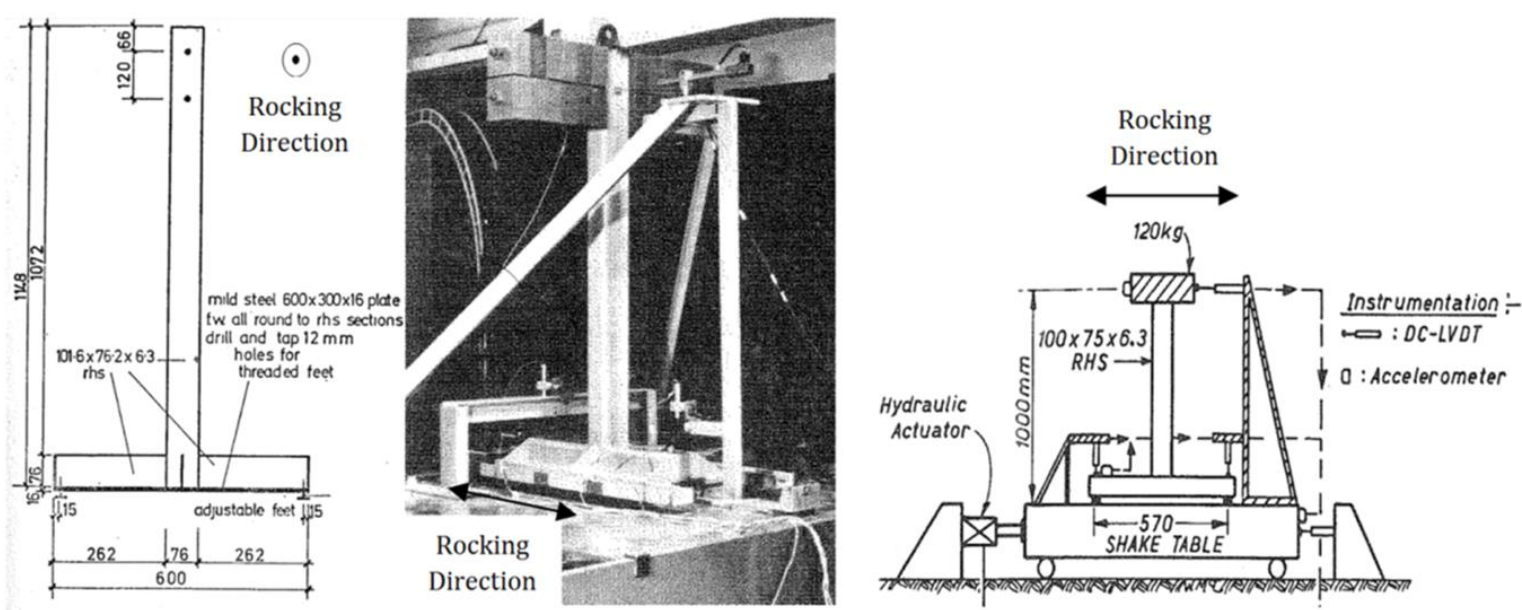

Figure 4: Experimental setup by Evison (figures from [4]): dimensions (left), photo (middle) and schematic of the shake table setup (right).

Evison [4] completed a number of free vibration tests. During these tests, the structure was displaced to an initial non-zero rocking angle and released. These tests allow examination of the influence of rocking action on vibrations without the influence of ground motion excitations. Figure 5 shows the recorded and simulated uplift displacement of the structure (top row), total horizontal acceleration (middle row) and total horizontal displacement at top mass level (bottom row) from a particular test. The experimental response was simulated by determining the initial rocking angle from the experimental data and assuming that the initial elastic displacement is equal to the critical displacement. The uplift displacement and total horizontal displacement traces from experiments and simulations shows excellent agreement for the first few cycles. Here, the reduction in the amplitude and duration of rocking cycles is well-captured. Notice that the uplift trace of both the 
experiment and simulation exhibit small high frequency oscillations. It is noteworthy that there is a slight time offset between uplift and horizontal displacement time histories. It is difficult to ascertain the cause of this offset, which may be attributed to unsimulated rigid body modes of the structure (e.g. sliding) or unaccounted errors during the data logging and digitization process.

The agreement between the experiment and simulations deteriorate as the specified impact model is not capable of detailed modelling of the energy dissipation mechanism. However, the overall agreement between simulations and the experiments is good, and this is further demonstrated by the acceleration and horizontal displacement traces. Similar to the uplift trace, the acceleration trace in Figure 5(middle row) reveals the presence of a significant high frequency oscillation with a frequency superposed on a step function. The oscillatory component is excited strongly at impact and is then damped throughout the rocking cycle. The simulation captures the general characteristics of these traces well, noting that the maximum acceleration estimates are conservative and the material damping factor $c_{r}$ can be improved for better time history descriptions.
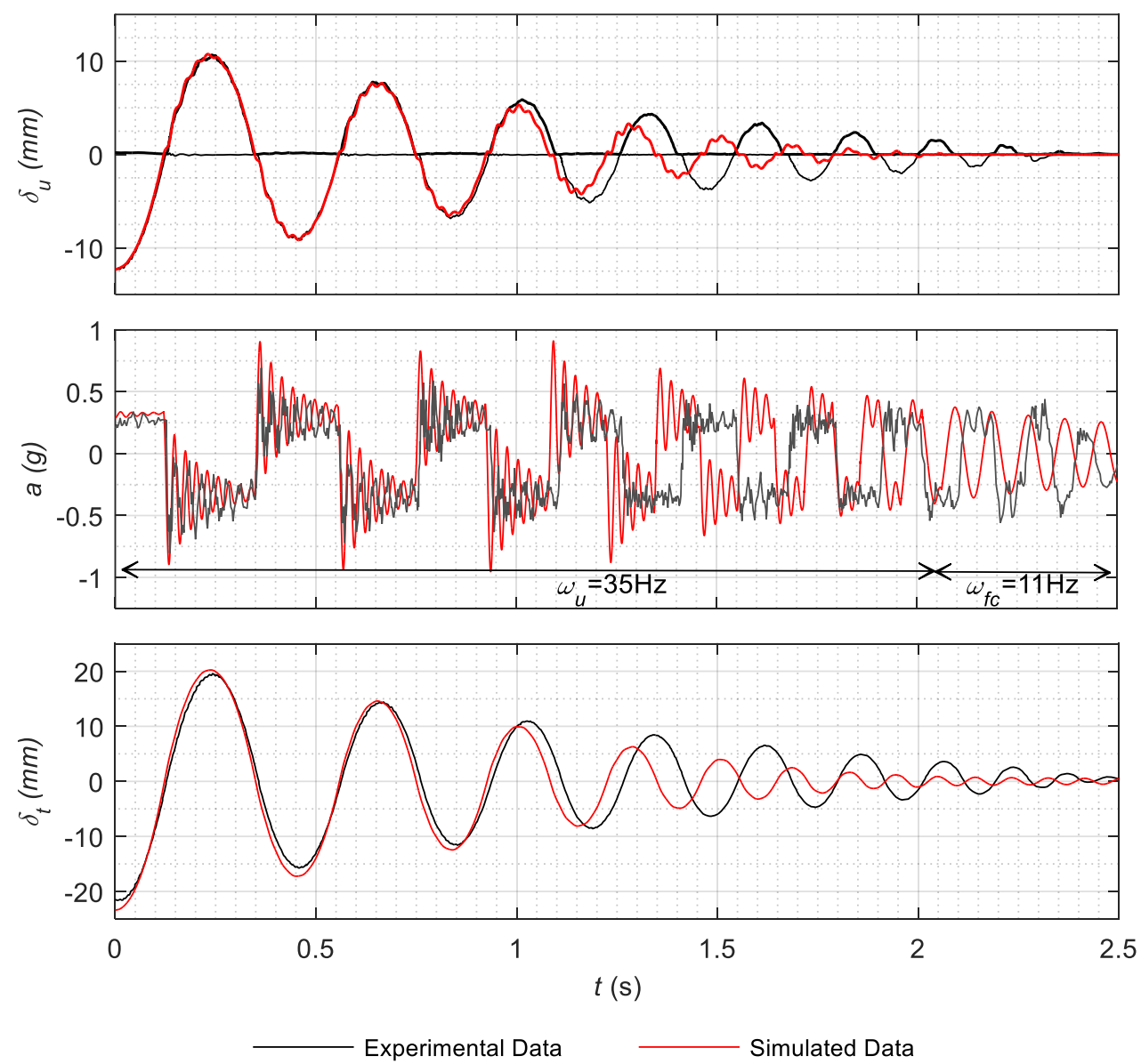

Figure 5: Recorded and simulated vertical uplift displacement trace (top row), lateral acceleration trace(middle row) and lateral displacement trace (bottom row) of the Evison [4] experimental specimen during a free vibration test.

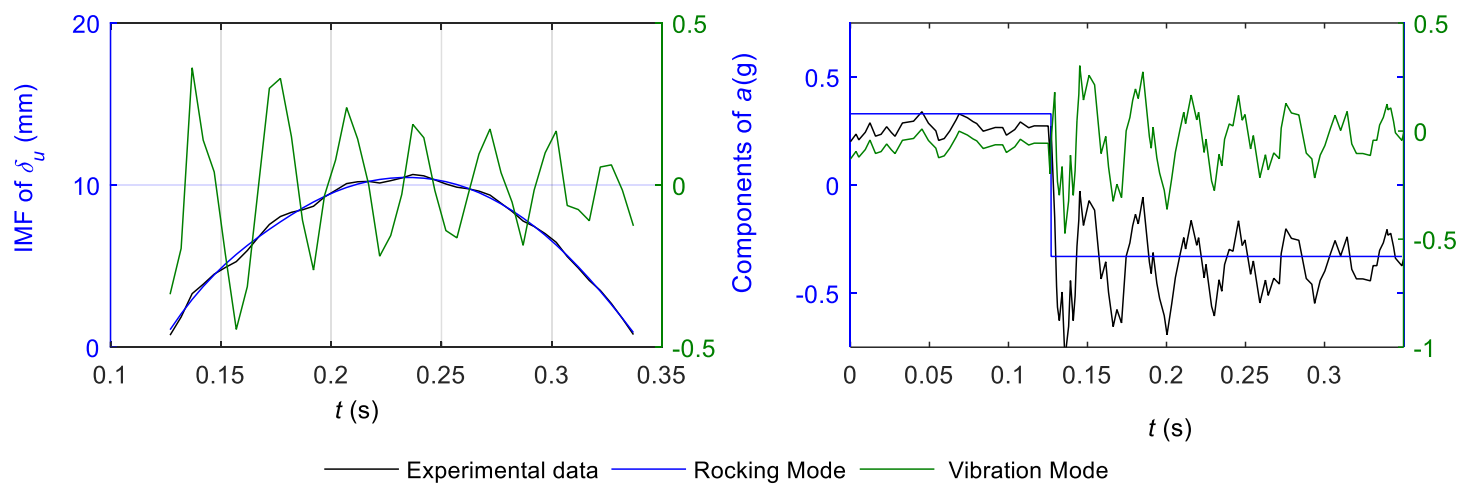

Figure 6: Modal components of the experimental uplift displacement (left) and acceleration trace (right) from a free vibration 
Having validated the simulations, it is useful to investigate the experimental data further to identify the modal components of response. The empirical modal decomposition [19] of the uplift trace of the rocking half-cycle after the first impact is presented in Figure 6 (left). The procedure separates the nonlinear uplift displacement signal into a smooth and an oscillatory component. These are the orthogonal rocking and vibration mode responses. It can be observed that the rocking motion due to the rocking mode is smooth and is responsible for the bulk of the rocking demand. On the contrary, the rocking motion due to the vibration mode is comparatively small and oscillatory. It demonstrates an approximate frequency of $35 \mathrm{~Hz}$, which is much higher than the experimentally determined vibration frequency during full contact of $11 \mathrm{~Hz}$.

The acceleration trace from the release of the structure until the second impact is also shown in Figure 6 (right). Similar to the uplift trace, the acceleration trace in Figure 6 reveals the presence of a significant high frequency oscillation superposed on a slowly changing function. The slowly changing function appears to have a near-constant value during both rocking cycles. This 'step' function is due to the rotational accelerations induced by the rocking mode and assumes near-constant values as the rocking angles are small. Therefore this acceleration demand due to the rocking may be estimated from Equation (13) as $0.34 \mathrm{~g}$. A comparison of this analytically identified rocking component (Figure 6, right) and the acceleration trace for the experiments suggest excellent agreement.

The second component of the acceleration trace in Figure 6 is the high frequency oscillation superposed on the step function. The cause of this oscillation was not identified by Evison [4]. However, the characteristics of this component of response strongly resemble the findings from Section 2. Equation (18) predicted that the vibration frequency during rocking phase would become $\omega_{u} \approx \omega_{f c} / \sqrt{1-\chi}=39 \mathrm{~Hz}$. This is similar to the experimentally observed vibration mode frequency of $35 \mathrm{~Hz}$ (Figure 5) where the signal frequency content is the largest. The oscillatory rocking action identified in Figure 6 (left) is also at this frequency, indicating that the vibrations induce rocking action as predicted by the model in Equation (16). In addition,
Equation (18) correctly predicts the strong excitation of vibrations at impact due to the change of direction of gravity forcing. There are no vibrations during the first rocking cycle where the structure is released from an initial displacement and then the vibrations get excited at impact. The maximum acceleration due to the vibration mode for the second rocking cycle is $0.48 \mathrm{~g}$. According to Equation (22), this indicates that the transition from one rocking phase to another was not instantaneous and the amplification factor was approximately $C=1.4$. This suggests that a gradual transition was observed between cycles, and limited the vibrations due to gravity forcing.

It was observed in Figure 5, that the lateral displacements of the structure were largely due to the rocking mode and that the rocking frequency depended on rocking amplitude. It is important to generalize this finding to determine the effective lateral frequency of the flexible rocking structure. The maximum horizontal displacement of the structure for a given rocking cycle at height $H$ is given by $\max \left(\delta_{t}\right)$. In Figure 7, $\max \left(\delta_{t}\right)$ is plotted against the effective rocking frequency of this motion $f_{t}$, which is calculated as the inverse of twice the time elapsed between zero crossings in the lateral displacement trace. In this figure, the frequency of rocking motion is also approximated by Equation (14). It is remarkable that the estimate for the effective vibration frequency of rocking mode, provided by Equation (13), adequately describes the frequency of horizontal motion of the experimental data for large displacements. This confirms that (i) the bulk of the rocking action is due to the rocking mode and (ii) the oscillatory rocking action due to vibration mode does not influence the effective period. However, the rocking frequency estimation of Equation (13) is less accurate for small displacements, especially when the horizontal displacement is less than the critical displacement. Equation (13) predicts increasing frequency of rocking, however, the response frequency of the structure remains close to the first mode frequency $\omega_{f c}=11 \mathrm{~Hz}$. In general, this figure provides guidance on how to estimate the effective lateral period of the structure using simple formulae.

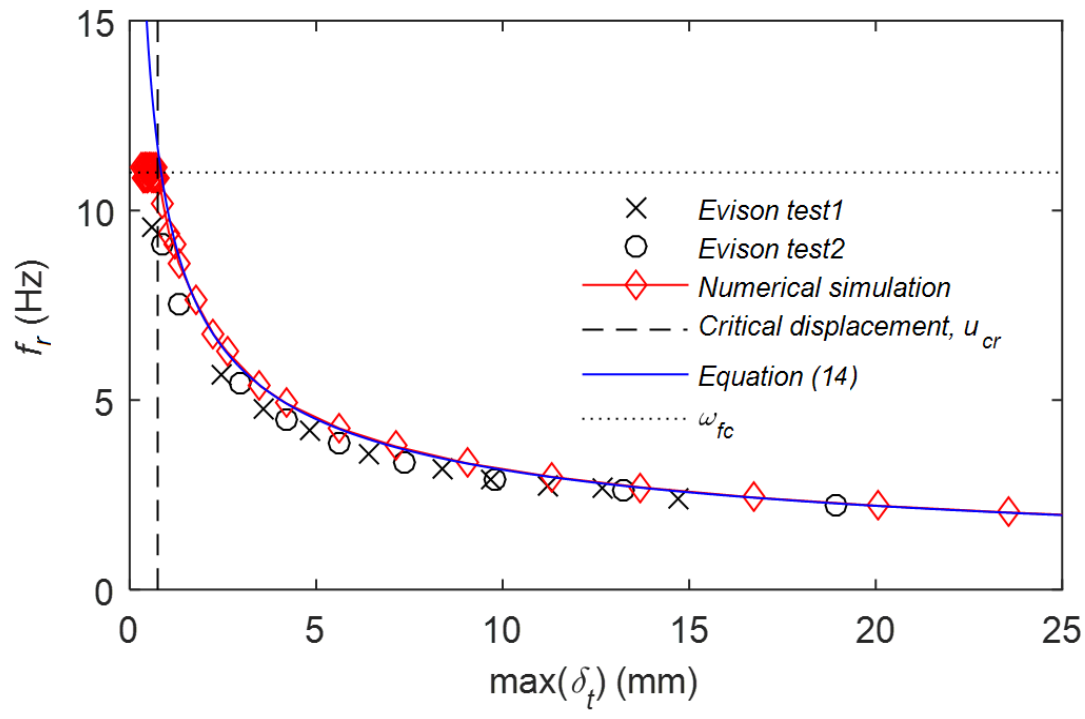

Figure 7: Recorded and simulated relationship between maximum horizontal displacement and the effective rocking frequency $f_{r}$, alongside simple theoretical estimates for the nonlinear relationship. 

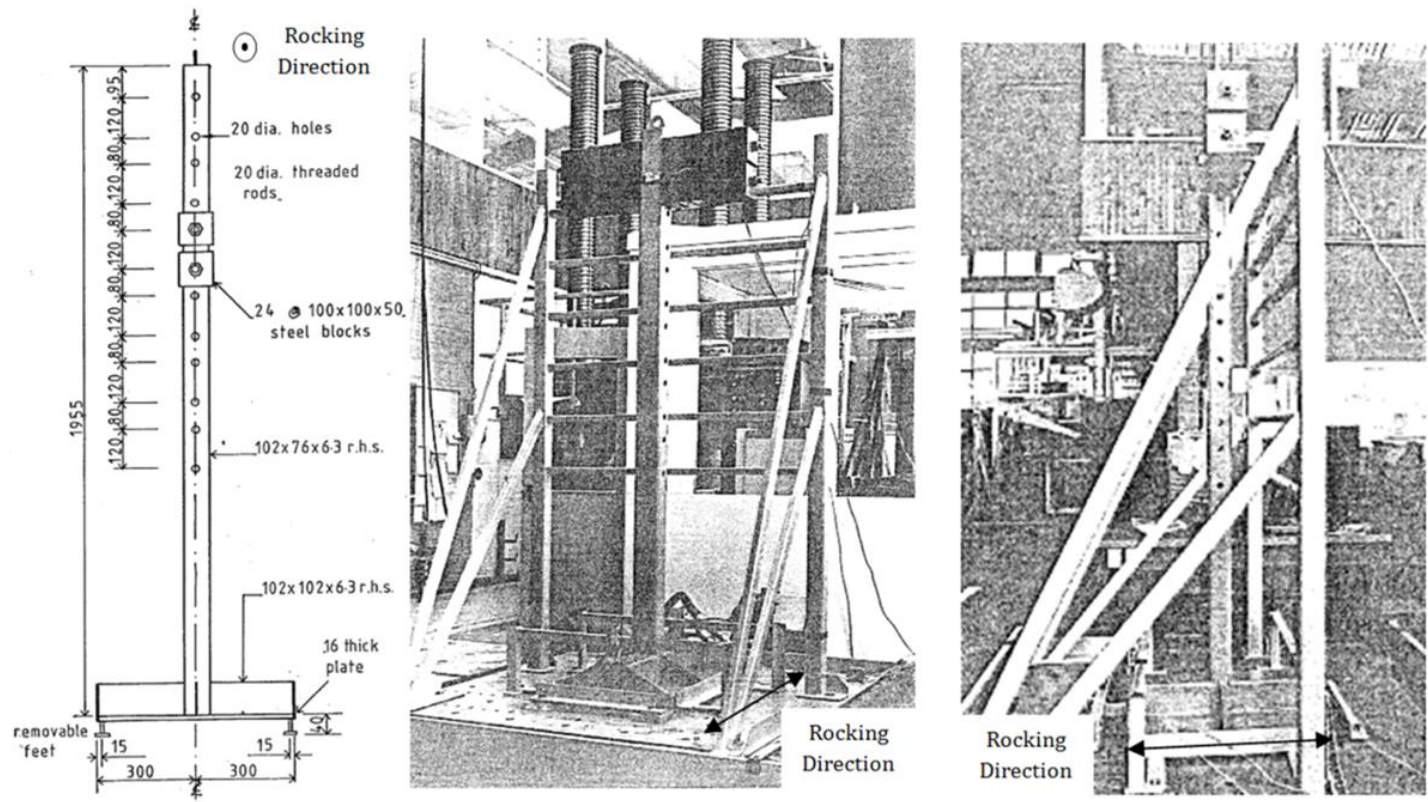

Figure 8: (left) A drawing illustrating the dimensions of the McManus experimental model with (middle)-(right) photos of the shake table setup of the model (Figures from: [5]).

Table 2: Important parameters for the analytical model representing the experimental model of McManus [5].

\begin{tabular}{|c|l|}
\hline Parameter & Value \\
\hline$B(\mathrm{~m})$ & 0.285 \\
\hline$H(\mathrm{~m})$ & 1.84 \\
\hline$m(\mathrm{~kg})$ & 130.1 \\
\hline$m_{0}(\mathrm{~kg})$ & 65.4 \\
\hline
\end{tabular}

\begin{tabular}{|c|l|}
\hline Parameter & Value \\
\hline$J_{0}\left(\mathrm{~kg} \cdot \mathrm{m}^{2}\right)$ & 10.7 \\
\hline$\omega_{f c}(\mathrm{~Hz})$ & 5 \\
\hline$\zeta_{f c}$ & $2 \%$ \\
\hline$r$ & 0.99 \\
\hline
\end{tabular}

\begin{tabular}{|c|l|}
\hline Parameter & Value \\
\hline$J_{\mathrm{P}}\left(\mathrm{kg} \cdot \mathrm{m}^{2}\right)$ & 467 \\
\hline$\alpha_{c g}(\mathrm{rad})$ & 0.23 \\
\hline$p_{l}(\mathrm{rad} / \mathrm{s})$ & 2.24 \\
\hline$\chi$ & 0.94 \\
\hline
\end{tabular}

To evaluate rocking isolation under the influence of an earthquake record, experimental results from McManus [5] are considered. The model utilized by McManus is similar to Evison [4], however, a more slender column where masses could be attached at various heights was utilized (see Figure 8). Tests with the $120 \mathrm{~kg}$ lumped at the mid-height of the structure were examined with flexible rocking models in a thorough study by Ma [20]. In this paper, the mass arrangement where the additional mass is lumped at the top are examined. The relevant details of this model are presented in Table 2. Shake table acceleration data is not provided by McManus [5], therefore the corresponding acceleration records from the PEER database were scaled to simulate the tests.

In Figure 9, alongside the scaled ground motion data used in simulations (top row), the uplift displacement (middle row) and total horizontal acceleration (bottom row) time histories from the experiments and the analytical simulations are presented. A coherent velocity pulse exists in the examined Bucharest and Pacoima Dam records, but it is superposed with high frequency acceleration components in the latter (see Figure 9, top row). The time and acceleration of the ground motion were scaled by a factor of 5 to satisfy similitude relationships [5]. Additional acceleration scaling factors of 0.35 and 0.29 were also specified for the Bucharest and Pacoima Dam earthquakes. In general, the reasonable prediction of the characteristics of the rocking and acceleration action validates the analytical model for earthquake excitation. The errors can be explained by simplifying assumptions regarding energy dissipation at impact and overestimation of viscous damping during rocking as well as potential differences in ground motion records used in the test and simulations.

To quantify the rocking isolation, the bottom row of Figure 9 shows the acceleration response of fixed base structures, with vibration frequency $\omega_{f c}$ and damping $\zeta_{f c}$, under the influence of the scaled Bucharest and Pacoima Dam records. Fixed base structures amplify the ground accelerations to $1.1 \mathrm{~g}$ and $2.1 \mathrm{~g}$, respectively, whereas the maximum accelerations experienced by the rocking structure are limited to $0.5 \mathrm{~g}$. Additionally, the acceleration trace during rocking cycles resembles acceleration traces from the free vibration tests in Figure 5. In both cases, the significant components of accelerations are due to the rocking mode and the gravity forcing of the vibration mode. The contribution of the direct excitation of the first vibration mode by the ground motion appears insignificant. This is also indicated by the proposed Equation (22), which provides a simple formula to assess the total lateral acceleration demand. The first term of Equation (22) provides a conservative estimate for the maximum vibration that can be directly excited by the ground motions, and indicates a value of $0.02 \mathrm{~g}$ and $0.10 \mathrm{~g}$ respectively for the Bucharest and Pacoima Dam earthquakes. On the other hand, the second term of Equation (22), describes the acceleration demands due to the rocking mode and excitation of vibrations at impact. In the absence of further information, an instantaneous transition is assumed between two rocking cycles, assuming a value of $C=2.0$. As a result, an upper bound acceleration demand of $0.66 \mathrm{~g}$ is obtained. It is clear that the latter term in Equation (22) is an order of magnitude larger. 

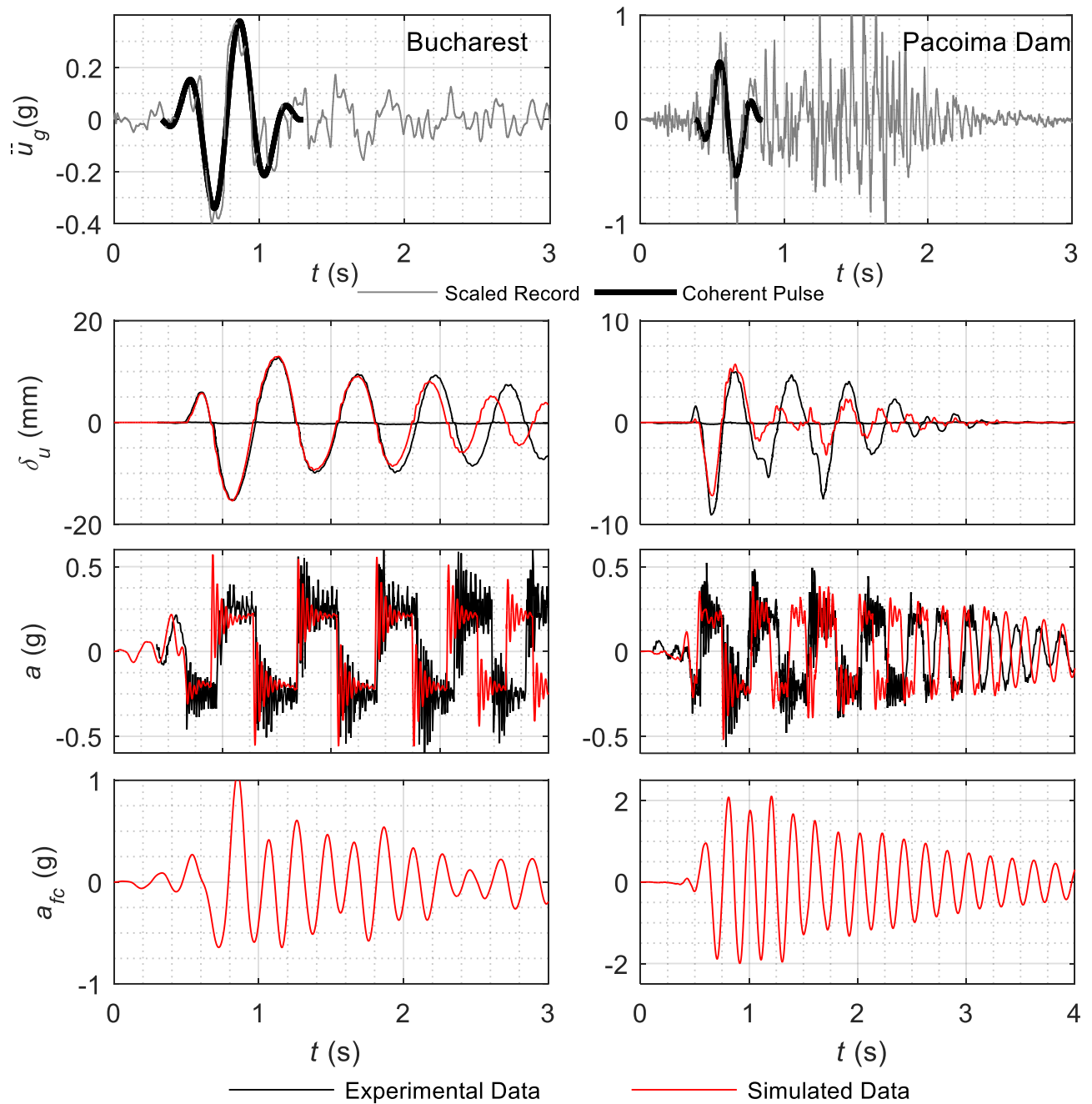

Figure 9: Simulation results for the Bucharest (left column) and Pacoima Dam (right column) shake table tests by McManus [5]: Scaled acceleration time histories used in the simulations $\left(1^{\text {st }}\right.$ row $)$, the comparison of experimental and analytical modeling of uplift ( $2^{\text {nd }}$ row) and horizontal acceleration traces ( $3^{\text {rd }}$ row), and the modelling of full contact acceleration response (4 $4^{\text {th }}$ row).

When $C=2.0$, Equation (22) predicts a conservative total acceleration demand of $0.68 \mathrm{~g}$ and $0.76 \mathrm{~g}$ for the Bucharest and Pacoima Dam earthquakes and captures the significance of the excitation of vibrations at impact. The measured maximum acceleration in both experiments is around $0.55 \mathrm{~g}$, which appears shortly after impact, without any correlation to particular ground movements. This observation indicates that calculating only the second term in Equation (22) may be sufficient to estimate the maximum acceleration demand for the first vibration mode of the structure. Additionally, it is useful to compare the $0.55 \mathrm{~g}$ maximum accelerations experienced by the McManus specimen to the corresponding $0.85 \mathrm{~g}$ measurement observed for Evison's model in free vibration tests (see Figure 5). This is due to the slenderness. As noted earlier, according to the second term of Equation (22), more slender structures are expected to incur lesser acceleration and force demands. These conclusions are supported by a recent study by the authors where they generalize the results of this study to complex multi-mass structures, where different vibration modes exist and are isolated to different degrees [15].

\section{CONCLUSIONS}

With the use of new analytical models and by revisiting some of the earliest tests on rocking structures by Evison [4] and McManus [5], this study re-evaluates the concept of rocking isolation. It demonstrates that the response of an idealized flexible rocking structure can be described as a superposition of a weakly coupled rocking mode and a coupled vibration mode.
The rocking mode exhibits the dynamic characteristics of a rigid structure and the force demands due to this mode are typically limited to those that are required to uplift the structure. An equation was proposed to estimate the effective frequency of the rocking mode, which is responsible for large displacement demands that are experienced during earthquakes. Then, vibration mode characteristics during rocking, which are poorly understood, were clarified. It was demonstrated that due to an effective decrease in mass participating in this mode, the vibration frequency increases. This change is accompanied by a number of interesting dynamic phenomena. Once rocking motion was initiated, the structural vibrations could only be weakly excited by the ground motion. However, due to the nonsmooth transition between rocking cycles, an instant reversal of gravity forces occurs. This induces significant vibrations in the superstructure. It was demonstrated that these vibrations due to gravity forcing are less significant for slender structures, and that they can be limited by gradual transitions between rocking cycles.

Based on these findings, equations were proposed for calculating maximum lateral acceleration, base shear and overturning moment demands for idealized flexible rocking structures. These equations were validated using the lateral acceleration traces from free vibration and earthquake excitation tests. They quantify the complex rocking isolation phenomena with simple equations and provide a useful basis to improve design methods (e.g. [8]) for estimating force demands in rocking structures. 


\section{REFERENCES}

1. Housner GW (1963). "Behaviour of inverted pendulum structures during earthquakes". Bulletin of the Seismological Society of America, 53(2): 403-417.

2. Beck JL and Skinner RI (1973). "The seismic response of a reinforced concrete bridge pier designed to step". Earthquake Engineering \& Structural Dynamics, 2(4): 343-358.

3. Sharpe RD and Skinner RI (1983). "The seismic design of an industrial chimney with rocking base". Bulletin of the New Zealand National Society for Earthquake Engineering, 16(2): 98-106.

4. Evison RJ (1977). "Rocking Foundations". Master Thesis. University of Canterbury, Canterbury.

5. McManus KJ (1980). "The Seismic Response of Bridge Structures Free to Rock on Their Foundations". Master Thesis, University of Canterbury, Canterbury.

6. Kelly JM and Tsztoo DF (1977). "Earthquake simulation testing of a stepping frame with energy-absorbing devices". Bulletin of the New Zealand National Society for Earthquake Engineering, 10(4): 196-207.

7. Skinner RI, Tyler RG, Heine AJ and Robinson WH (1980) "Hysteretic dampers for the protection of structures from earthquakes". Bulletin of the New Zealand National Society for Earthquake Engineering, 13(1): 22-36.

8. Kelly TE (2009). "Tentative seismic design guidelines for rocking structures". Bulletin of the New Zealand National Society for Earthquake Engineering, 42(4): 239-274.

9. Acikgoz S and DeJong MJ (2012). "The interaction of elasticity and rocking in flexible structures allowed to uplift". Earthquake Engineering \& Structural Dynamics, 41(15): 2177-2194.

10. Acikgoz S and DeJong MJ (2013). "The rocking response of large flexible structures to earthquakes." Bulletin of Earthquake Engineering, 12(2): 875-908.
11. Meek JW (1975). "Effects of foundation tipping on dynamic response". Journal of the Structural Division, 101(7): 1297-1311.

12. Chopra AK and Yim CS (1985). "Simplified earthquake analysis of structures with foundation uplift". Journal of Structural Engineering, 111(12): 2708-2731.

13. Acikgoz S and DeJong MJ (2016). "Analytical modelling of multi-mass flexible rocking structures". Earthquake Engineering \& Structural Dynamics, 45(13): 2103-2122.

14. Acikgoz S, Ma QT, Palermo A and DeJong MJ (2016). "Experimental identification of the dynamic characteristics of a flexible rocking structure." Journal of Earthquake Engineering, 20(8): 1-23.

15. Acikgoz S and DeJong MJ (2016). "Vibration modes and equivalent models for flexible rocking structures". Bulletin of Earthquake Engineering, In Press.

16. Giouvanidis AI and Dimitrakopoulos EG (2016). "Nonsmooth dynamic analysis of sticking impacts in rocking structures". Bulletin of Earthquake Engineering, In Press.

17. Truniger R, Vassiliou MF and Stojadinovic B (2015). "An analytical model of a deformable cantilever structure rocking on a rigid surface: experimental validation". Earthquake Engineering \& Structural Dynamics, 44(15): 2795-2815.

18. ElGawady MA, Ma QT, Butterworth JW and Ingham JM (2010). "Effects of interface material on the performance of free rocking blocks". Earthquake Engineering \& Structural Dynamics, 40(4): 375-392.

19. Huang NE, Shen Z, Long SR, Wu MC, Shih HH, Zheng Q Yen NC, Tung CC and Liu HH (1998). "The empirical mode decomposition and the Hilbert spectrum for nonlinear and non-stationary time series analysis". Proceedings of the Royal Society of London Series A: Mathematical, Physical, and Engineering Sciences, 454(1971): 903-995.

20. Ma QT (2010). "Mechanics of Rocking Structures Subjected to Ground Motion". PhD Thesis. University of Auckland, Auckland. 